\title{
Probing the Kinematics of the Narrow-Line Region in Seyfert Galaxies with Slitless Spectroscopy: Observational Results ${ }^{1}$
}

\author{
J.R. Ruiz ${ }^{2}$, D.M. Crenshaw ${ }^{3}$, S.B. Kraemer ${ }^{2}$, G.A. Bower ${ }^{4}$, T.R. Gull ${ }^{5}$, J.B. Hutchings ${ }^{6}$, \\ M.E. Kaiser ${ }^{7}, \&$ D. Weistrop ${ }^{8}$
}

\begin{abstract}
We present slitless spectra of 10 Seyfert galaxies observed with the Space Telescope Imaging Spectrograph on the Hubble Space Telescope (HST). The spectra cover the $[\mathrm{O}$ III $] \lambda \lambda 4959,5007$ emission lines at a spectral resolving power of $\lambda / \Delta \lambda \approx 9000$ and a spatial resolution of $\sim 0^{\prime \prime} .1$. We compare the slitless spectra with previous $H S T$ narrow-band images to determine the velocity shifts and dispersions of the bright emission-line knots in the narrow-line regions (NLRs) of these Seyferts, which extend out to at least several hundred pc from their nuclei. Many knots are spatially resolved with sizes of tenths of arcsecs, corresponding to tens of pcs, and yet they appear to move coherently with radial velocities between zero and $\pm 1200 \mathrm{~km} \mathrm{~s}^{-1}$ with respect to the systemic velocities of their host galaxies. The knots also show a broad range in velocity dispersion, ranging
\end{abstract}

\footnotetext{
${ }^{1}$ Based on observations made with the NASA/ESA Hubble Space Telescope, obtained at the Space Telescope Science Institute, which is operated by the Association of Universities for Research in Astronomy, Inc., under NASA contract NAS 5-26555. These observations are associated with proposal GO-8340.

${ }^{2}$ Catholic University of America and Laboratory for Astronomy and Solar Physics, NASA's Goddard Space Flight Center, Code 681, Greenbelt, MD 20771; ruiz@yancey.gsfc.nasa.gov

${ }^{3}$ Department of Physics and Astronomy, Georgia State University, Astronomy Offices, One Park Place South SE, Suite 700, Atlanta, GA 30303; crenshaw@chara.gsu.edu

${ }^{4}$ Computer Sciences Corporation, Space Telescope Science Institute, 3700 San Martin Drive, Baltimore, MD 21218

${ }^{5}$ Laboratory for Astronomy and Solar Physics, NASA's Goddard Space Flight Center, Code 681, Greenbelt, MD 20771

${ }^{6}$ Dominion Astrophysical Observatory, National Research Council of Canada, 5071 West Saanich Rd., Victoria, BC V9E 2E7, Canada

${ }^{7}$ Department of Physics \& Astronomy, Johns Hopkins University, 3400 North Charles St., Baltimore, MD 21218

${ }^{8}$ Department of Physics, University of Nevada at Las Vegas, 4505 Maryland Parkway, Las Vegas, NV 89154-4002
} 
from $\sim 30 \mathrm{~km} \mathrm{~s}^{-1}$ (the velocity resolution) to $\sim 1000 \mathrm{~km} \mathrm{~s}^{-1}$ FWHM. Most of the Seyfert galaxies in this sample show an organized flow pattern, with radial velocities near zero at the nucleus (defined by the optical continuum peak) and increasing to maximum blueshifts and redshifts within $\sim 1^{\prime \prime}$ of the nucleus, followed by a decline to the systemic velocity. However, there are large local variations around this pattern and in one case (NGC 7212), the radial velocities are nearly chaotic. The emission-line knots also follow a general trend of decreasing velocity dispersion with increasing distance from the nucleus. In the Seyfert 2 galaxies, the presence of blueshifts and redshifts on either side of the nucleus indicates that rotation alone cannot explain the observed radial velocities. The most straightforward interpretation is that radial outflow plays an important role in the NLR kinematics. Each of the Seyfert galaxies in this sample (with the exception of Mrk 3) shows a bright, compact (FWHM $\left.\leq 0^{\prime \prime} .5\right)$ [O III] knot at the position of its optical nucleus. These nuclear emission-line knots have radial-velocity centroids near zero, but they typically have the highest velocity dispersions. Their similar properties suggest they may be a common, distinct component of the NLR.

Subject headings: galaxies: Seyfert

\section{Introduction}

The narrow-line region (NLR) in Seyfert galaxies is the smallest resolvable structure in the UV and optical that is directly affected by the ionizing radiation and dynamical forces from the inner active nucleus. Hence, studies of the physical conditions and kinematics of the NLR should yield important insights into the machinery of active galactic nuclei (AGN), as material is processed into and out of the environment surrounding the central supermassive black hole $(\mathrm{SMBH})$. The NLR also provides information on the interactions between the host galaxy and the inner unresolved nucleus, which is thought to include a central SMBH, accretion disk, broad-line region (BLR), and dusty molecular torus (see Jaffe et al. 2004). By definition, the NLR is responsible for narrow emission lines with widths $\approx 500 \mathrm{~km} \mathrm{~s}^{-1}$ full-width at half-maximum (FWHM), whereas emission lines from the BLR have widths in the range $1000-7000 \mathrm{~km} \mathrm{~s}^{-1}$ FWHM. Also by definition, Seyfert 2 galaxies show only narrow emission lines in their UV and optical spectra, while Seyfert 1 galaxies show both broad and narrow emission lines. Whereas the BLR has a size of only tens of light days (Peterson et al. 2004), the NLR extends out to at least several hundred pcs from the nucleus 
(Schmitt et al. 2003a, b). Nevertheless, the angular sizes of the NLRs in nearby Seyfert galaxies are still quite small; for example, at a redshift of 0.01 and $\mathrm{H}_{0}=75 \mathrm{~km} \mathrm{~s}^{-1} \mathrm{Mpc}^{-1}$, $100 \mathrm{pc}$ subtends only $\sim 0^{\prime \prime} .5$. Thus, detailed studies of the physical conditions and kinematics across the NLR were not possible before the advent of the Hubble Space Telescope (HST) and ground-based adaptive optics.

HST has provided emission-line images of the NLRs in many nearby Seyfert galaxies at a spatial resolution of $\sim 0^{\prime \prime} .1$. The images show that NLRs tend to be clumpy, with many resolved "knots" of emission that are typically a few tenths of an arcsec in size (Evans et al. 1991, 1993; Schmitt et al. 2003a). The overall morphology of the NLR is varied; triangular (or "fan-like"), linear, spiral (or "S" shaped), elliptical, circular, arc-like, and amorphous shapes are all common. However, there is a trend in the sense that the NLRs in Seyfert 2 galaxies are more elongated than those in Seyfert 1 galaxies, which tend to be more concentrated and circular in appearance (Schmitt et al. 2003b) This result is in qualitative agreement with the unified model (Antonucci 1993), which posits that the two types of Seyfert galaxies are due to our viewing angle with respect to an optically thick torus outside of the BLR, which produces bicones of ionizing radiation in the NLR. In the case of a Seyfert 2 galaxy, the torus blocks our view of the BLR, and we see a more extended, triangular NLR. For a Seyfert 1 galaxy, we are looking down the cone to see the BLR, and the NLR looks more compact and circular ${ }^{1}$. The triangular-shaped NLRs seen in some Seyfert 2 galaxies are clear examples of projected bicones (with one of the cones diminished or extinguished in some cases by the host galactic plane); other NLR morphologies could be explained by selective filling of the ionization bicones by material in ways that are admittedly not well understood.

The physical conditions in the NLR have been studied extensively, and it is clear that photoionization by the central continuum source (the accretion disk) plays a dominant role in heating the gas, although shocks may play an important role in localized regions (Kraemer \& Crenshaw 2000; Dopita et al. 2002). The dynamics of the gas in the NLR, however, are less certain. Prior to $H S T$, most studies had to rely on spatially-integrated line profiles. Unfortunately, the profiles can be reproduced by a wide variety of kinematic models (e.g., infall, outflow, rotation, parabolic orbits, etc., (e.g., Osterbrock \& Mathews, 1986, and references therein; De Robertis \& Shaw 1990; Veilleux 1991; Moore \& Cohen 1996). Thus, spatially-resolved spectra across the NLR are essential for further kinematic constraints. Early attempts with HST made use of the long-slit spectroscopy mode of the Faint Object Camera (FOC), resulting in kinematic information on the NLRs in NGC 4151 (Winge et al.

\footnotetext{
${ }^{1}$ Two notable exceptions are the Seyfert 1 galaxies NGC 4151 (Evans et al. 1993) and NGC 3516 (Ferruit et al. 1998), which show elongated NLRs and are likely viewed at intermediate angles.
} 
1997; 1999), NGC 1068 (Axon et al. 1998), and Mrk 3 (Capetti et al. 1999). Several models were advocated based on these data, including rotation around the SMBH and interior mass, radial acceleration by radio jets, and expansion of "hot cocoons" of gas around the jets, or more accurately, radio lobes, forcing the NLR clouds away from the lobes (Axon et al. 1998; Capetti et al. 1999).

The installation of the Space Telescope Imaging Spectrograph (STIS) on HST in 1997 February provided an ideal opportunity for detailed kinematic mapping of the NLR. Using STIS long-slit and slitless observations, we presented evidence that radial outflow is the dominant component of motion in the NLRs of three Seyfert galaxies: NGC 4151 (Hutchings et al. 1998; Kaiser et al. 2000; Nelson et al. 2000; Crenshaw et al. 2000), NGC 1068 (Crenshaw \& Kraemer 2000), and Mrk 3 (Ruiz et al. 2001). We found no connection between the kinematics of the NLR clouds and the radio lobes (Kaiser et al. 2000; Ruiz et al. 2001), and concluded that the outflow was more likely the result of radiation pressure or winds than radio jets. Comparison of the radial-velocity fields in these three Seyferts with biconical outflow models indicated that the NLR gas accelerates outward from the nucleus to a turnover point, at a distance of $\sim 100 \mathrm{pc}$ from the nucleus, and subsequently appears to decelerate to the systemic velocity at a distance of $\sim 300 \mathrm{pc}$. At larger distances, the velocities are consistent with rotation in the galactic plane, which agrees with results from earlier ground-based studies of extended narrow-line regions (ENLRs), located at distances greater than a few hundred parsecs from the nucleus (Unger et al. 1987).

To expand the collection of Seyfert galaxies with NLR kinematic data, we have obtained STIS slitless spectra of 10 Seyfert galaxies at high spectral resolution. The principal advantage of the slitless technique is that it is very efficient: the radial velocities of all of the bright knots in the NLR can be mapped with a single pointing of HST. The principal disadvantage, compared to long-slit observations, is that faint knots or more diffuse emission cannot be detected and measured with this technique. Thus, the sample is biased, in the sense that we picked Seyfert galaxies with NLRs that are known to be extended and have high-contrast knots, based on existing HST [O III] images. In this paper, we present the observational results for our slitless program. Some of the results for Mrk 3 have already been published (Ruiz et al. 2001); we include it in this paper for completeness. In a subsequent paper, we discuss our comparison of the observations with kinematic models (D.M. Crenshaw et al. 2004, in preparation). 


\section{Observations and Analysis}

\subsection{Sample and Technique}

The basic idea behind the slitless technique is to measure the displacement of an emission-line knot, originally detected in a narrow-band image, after a grating is introduced. The displacement in the dispersion direction yields the average radial velocity of the knot, and the broadening of the emission gives the velocity dispersion of the knot. To further increase the efficiency of this program, we chose Seyfert galaxies with existing HST [O III] images, which are needed to determine the spatial locations of the knots on the sky. We have used this technique successfully in the past, with STIS slitless spectra of NGC 4151 (Hutchings et al. 1999; Kaiser et al. 2000). To demonstrate the reliability of this technique, we compared the radial velocities from STIS slitless and long-slit observations of NGC 4151 (Crenshaw et al. 2000), and found they differ by only $\pm 50 \mathrm{~km} \mathrm{~s}^{-1}$ on average, whereas the range of radial velocities for the bright knots is on the order of $\pm 800 \mathrm{~km} \mathrm{~s}^{-1}$.

Table 1 lists our sample, which includes eight Seyfert 2, one Seyfert 1.9 (Osterbrock 1981), and one Seyfert 1 galaxy. The table also gives the host galaxy types, redshifts of the host galaxies (from stellar absorption lines when H I 21-cm observations are not available), and our adjustments to the redshifts based on measured ENLR velocities, as described in §2.3. Previous HST narrow-band images showed that all of these NLRs have extended, high-contrast knots of [O III] emission.

We obtained new observations of each Seyfert galaxy with the STIS CCD detector, G430M grating, and an open aperture $\left(52^{\prime \prime} \times 52^{\prime \prime}\right)$. The CCD is a $1024 \times 1024$ pixel detector with a plate scale of $0^{\prime \prime} .051$ pixel $^{-1}$ and an angular resolution of $\sim 0^{\prime \prime} .1 \mathrm{FWHM}$ in imaging mode. With the G430M grating in place, the scale is changed to $0^{\prime \prime} .057$ pixel $^{-1}$ in the dispersion direction due to anamorphic magnification. For a point source, the dispersion of the G430M grating is $0.28 \AA$ pixel $^{-1}$, and the spectral resolution is twice this value, which yields a resolving power of $\sim 9000$ and a velocity resolution of $\sim 33 \mathrm{~km} \mathrm{~s}^{-1}$ (FWHM) in the vicinity of the [O III] $\lambda 5007$ line. For the extended emission-line knots, the resolution is degraded, but most have angular sizes $\leq 0^{\prime \prime} .3$ and velocity widths $>100 \mathrm{~km} \mathrm{~s}^{-1} \mathrm{FWHM}$, so this has little effect on our measurements. Since the NLRs are all much smaller than the CCD field of view and we did not use a slit, we obtained the observations without target acquisition, to maximize the exposure time in an orbit. We observed each target for a single orbit.

Table 2 gives a log of our STIS grating observations, which includes the STScI dataset names, UT dates of observation, central wavelength settings, total exposure times, and position angles (from north in an eastward direction) of the Y-axis of the CCD chip (which 
is perpendicular to the dispersion direction). The wavelength coverage of the G430M grating is only $286 \AA$, so we chose the central wavelength such that the redshifted [O III] $\lambda 5007$ line was well covered. This resulted in coverage of the [O III] $\lambda 4959$ line in all cases, and the $\mathrm{H} \beta$ line in five cases. The listed exposure time is the sum from two separate, equal exposures obtained in the orbit for the purpose of eliminating cosmic-ray hits. The position angle was chosen to place the minor axis of the NLR emission roughly along the STIS dispersion axis, to minimize confusion from overlapping clouds in the dispersion direction.

To measure the radial velocities of the emission-line knots, we used four images of each Seyfert galaxy: a direct continuum and an emission-line image from the STScI/MAST archives, a STIS direct broad-band image, and the STIS spectral image. In addition, we obtained two calibration images at each pointing, as discussed below. Ideally, one would like to determine the radial velocities of the knots in a slitless spectrum by comparing it with a direct [O III] image obtained with the same instrument and orientation. However, given the redshifts of these Seyfert galaxies, STIS does not have suitable [O III] filters for this purpose. Thus, we relied on archival HST emission-line and continuum images obtained with the WFPC2, FOC, and WF/PC (PC mode) cameras, in order of preference. However, we still required a direct STIS image to properly register the archival and STIS spectral images, since most of our targets are Seyfert 2 galaxies, and lack a bright central point-source (like that in NGC 4151), which is convenient for alignment purposes. Thus, we obtained a short (20 s) direct exposure in STIS camera mode, which is sensitive from $2000-10,000 \AA$ and is dominated by continuum emission, but contains line emission as well. We rotated the archival continuum image using the position angle in the header and aligned it with the STIS direct image using common features, particularly the central continuum peak (due to the AGN in NGC 3516 and stellar emission in the Seyfert 1.9 and 2 galaxies). This also resulted in alignment of the archival [O III] image with the direct STIS image, since it was obtained during the same pointing as the archival continuum image. In a number of instances, we were also able to check our alignment by comparing emission-line knots detected in the STIS direct image with those in the archival [O III] images.

A final translation of all of the direct images was needed in the STIS dispersion direction to align them with the STIS spectral image, by placing the emission-line knots at their expected locations if they had zero radial velocity with respect to the systemic redshift of the host galaxy. This was done with the help of two calibration images obtained at each pointing: a direct image of a continuum lamp through a $52^{\prime \prime} \times 0^{\prime \prime} .1$ slit, and a wavelength calibration image of an emission-line lamp with the G430M grating and the same slit. The displacement in the dispersion direction between the continuum-lamp image and the expected position of redshifted [O III] $\lambda 5007$ (from the redshifts in Table 1) gives the shift needed to align the direct and spectral images, such that the measured displacement for a knot gives 
the radial velocity with respect to systemic. This final step is an improvement over the

procedure used for NGC 4151 (Kaiser et al. 2000), in that we are able to determine absolute radial velocities as opposed to relative values (in that case, with respect to the bright central knot of [O III] emission). The final alignment, rotation to the position angle of the STIS images, rebinning to the STIS plate scale, and translation in the dispersion direction were actually accomplished with a single resampling of the archival images.

Table 3 gives the archival images used for this study. Most of these images have been discussed in detail by other authors. The emission-line (narrow-band) images are those with an "N" suffix, whereas the continuum images (medium- or wide-band) images are those with "M" or "W" suffixes. The FOC narrow-band image of Mrk 3 is shown in Ruiz et al. (2001), and was useful for detecting extended emission. Mrk 573 has continuum and emission-line images obtained at different times, but we were able to align these images using common features.

\subsection{Direct and Dispersed Images}

We use the images of Mrk 3 to illustrate the general effects of the filters and grating used for this study. In Figure 1, we show three archival images of Mrk 3, along with the STIS spectral image on the same scale. In the F502N image, the [O III] emission dominates in the form of bright knots of emission in a backwards "S" shape. However stellar continuum emission is also clearly seen in the form of a compact circular shape, which dominates the emission in the F547M continuum image. The F606W filter is broad enough to contain substantial emission from both continuum and emission-lines, and deep enough to show some of the extended [O III] emission in a biconical shape. The STIS slitless spectrum shows all of these features: the bright central knots show large displacements due to their radial velocities and are highly broadened as a result of their velocity dispersions. The continuum is faintly visible as a broad horizontal band; the clouds in the ENLR, at distances $\gtrsim 1^{\prime \prime} .5$ from the nucleus, have substantially lower radial velocities and velocity widths than those in the NLR (see also Ruiz et al. 2001).

Figure 2 displays side-by-side [O III] and STIS slitless images of the remaining nine Seyfert galaxies in our sample. The [O III] images are not corrected for continuum emission, in order to show the effects of this emission in the STIS spectra. Distortions in the spectral images, compared to the direct images, are due to radial-velocity shifts and velocity broadening of the emission-line knots. The velocity broadening is most apparent in the inner NLRs, typically within $\sim 1^{\prime \prime}$ of the optical continuum peaks. It is clear from the images that most of the emission-line clouds seen in the direct images can be identified without difficulty 
in the spectral images.

The broad horizontal bands in the STIS spectra near the galaxy nuclei, extending over $1^{\prime \prime}-5^{\prime \prime}$, are primarily due to stellar continuum emission in the host galaxy (the narrow bands offset from the nuclei are individual stars in the STIS field of view). Most of the structures in the continuum bands are due to dust lanes, which can be seen in the archival continuum images (e.g., Malkan, Gorjian, \& Tam 1998). We have checked for scattered light from the hidden nucleus, by extracting regions of enhanced continuum emission and looking for broad $\mathrm{H} \beta$ emission in the five Seyfert 2 galaxies with coverage of this line (Mrk 620, NGC 1386, NGC 5506, NGC 5643, and NGC 7212). No broad H $\beta$ emission was detected. However, slitless spectra are not optimal for detecting scattering regions, since their emission would be convolved with other regions of pure stellar emission in the dispersion direction. Thus we cannot rule out local regions of scattered emission in these five Seyfert galaxies, such as those detected in STIS long-slit spectra of NGC 1068 on scales of $\sim 0^{\prime \prime} .3$ (Crenshaw \& Kraemer 2000).

Perhaps the most striking feature in the images in Figure 2 is the bright, central knot of [O III] emission in each object, which is highly dispersed in the STIS images. In each case, it is located at the optical continuum peak (indicated by a "+"). These knots are spatially resolved in most cases, with sizes that range from $0 " .15$ to 0 ". 5 FWHM perpendicular to the STIS dispersion. As shown in $§ 2.3$, the nuclear knots typically have the highest velocity dispersions, but have radial-velocity centroids that tend to be close to zero.

There are a few images that deserve further comment at this point. The FR533N image of Mrk 620 shows an extended ring of emission that is not apparent in the STIS [O III] line, but is clearly seen in the STIS $\mathrm{H} \beta$ line. The high $\mathrm{H} \beta /[\mathrm{O}$ III $]$ ratio in this ring indicates lowionization gas that is likely due to starburst activity. However, the small V-shaped feature extending toward the east is [O III] emission from higher-ionization gas that is presumably directly ionized by the AGN. The F501N image of NGC 5506 only covers one side of the NLR (a clear example of a cone in projection), whereas the STIS spectral image shows a faint counter-cone south of the optical continuum peak; we cannot measure the radial velocities of the knots in this region without a comparison image. There is no narrow-band image of NGC 5728, but the medium-band F492M image shown in Figure 2c contains [O III] emission that we can use for registration of the emission-line knots. This filter transmits substantially more continuum than the narrow-band filters, which explains the elliptical disk of continuum emission that shows up as a broad horizontal band in the STIS spectrum. 


\subsection{Measurements}

We processed the STIS spectra with the IDL software developed at NASA's Goddard Space Flight Center for the Instrument Definition Team. We removed cosmic-ray hits using the two exposures of each target, and removed hot or warm pixels by interpolation in the dispersion direction. We used the calibration exposures described in $\S 2.1$ to obtain a wavelength scale and processed the spectral images in "extended" mode, which geometrically rectifies the images to produce a constant wavelength along each column and fluxes at each position in units of ergs s${ }^{-1} \mathrm{~cm}^{-2} \AA^{-1}$ per pixel (fluxes are conserved in the geometric transformation).

The challenge of slitless spectroscopy is to identify an emission-line knot, displaced by the introduction of a grating, in both the direct and dispersed images. As a guide, we utilized the fact that a given knot's location and extent perpendicular to the dispersion are the same in the two images. Once we identified the knots, we fit the spatial and spectral images row-by-row (parallel to the dispersion and separated by $0{ }^{\prime \prime} .05$ intervals) with a Gaussian for each knot plus a straight line for the background emission. We then determined the radial velocity of each knot by subtracting the positions of the Gaussian peaks in the direct and dispersed images, and the intrinsic velocity width by subtracting the FWHMs of the two Gaussians in quadrature. In the few instances where the FWHM was slightly smaller in the dispersed image, due to statistical fluctuations, we set the intrinsic FWHM to zero. We did not average together values in different rows, so we have multiple measurements of each knot, the number of which depends on its extent in the spatial direction of the dispersed image. Occasionally, a single knot in the direct image would split into two knots in the dispersed image, indicating two knots with different radial velocities that appear as one in projection.

After determining the radial velocities as a function of position, it became evident that some of the galaxies showed systematic offsets from zero in the ENLR on both sides of the nucleus. Given the available evidence that the ENLR lies in the host galaxy, we added a constant to the radial velocities of galaxies for which the average offset was $>50 \mathrm{~km} \mathrm{~s}^{-1}$ (the approximate measurement error) to bring the average radial velocity in the ENLR to zero. In some cases, there are still asymmetries in the ENLR velocities on either side of the nucleus, which can be ascribed to galactic rotation. The velocity offsets are given in Table 1. The systematic errors in radial velocity could be the result of small misregistrations among the various images and/or inaccurate values for the systemic velocities. The latter might

explain why Mrk 573 has such a large correction; it is one of only two galaxies for which we had to rely on stellar absorption lines for its systemic redshift. 


\section{Results}

Most of our analysis focuses on radial velocities and FHWMs as functions of projected angular distance from the optical continuum peak (which we refer to as the "nucleus"). The slitless spectra can also be used to look for variations in these quantities as a function of distance from the major axis of the NLR, or as a function of position angle with respect to the major axis, but we found no convincing evidence for such variations. It is possible that they are present, but are overwhelmed by strong changes in the velocities as a function of radial distance from the nucleus. Since our coverage is limited to only the bright knots of emission, more detailed maps of the NLR kinematics, using multiple slit positions at high spectral resolution (e.g., Cecil et al. 2002), are required to investigate the above, more subtle, effects.

In Figure 3, we show the measured radial velocities and FWHMs for Mrk 3 as a function of projected angular distance from the nucleus on the sky. The trend in radial velocities agrees well with our previous measurements from long-slit, low-resolution spectra and from the slitless spectrum in which we averaged the velocities for each knot (Ruiz et al. 2001). In fact, individual knots can be identified as points clumped together in the radial-velocity plot. The advantage of long-slit observations can be seen in Figure 8 of Ruiz et al. (2001), which shows a much cleaner trend due to the ability to measure fainter emission along the slit. The overall trend is an increase in radial velocity from near zero at the nucleus to maximum blueshifts and redshifts at $\sim 0^{\prime \prime} .3$ on either side of the nucleus, followed by a decline to the systemic velocity (with large scatter in Figure 3 ) at distances $\gtrsim 2^{\prime \prime}$. The FWHM plot in Figure 3 shows large scatter at each location as well, but there is a trend of higher velocity dispersions for the bright inner clouds, as can be seen in Figure 1.

We show radial velocity and FWHM plots for the other nine Seyfert galaxies in Figure 4. Row-by-row measurements of each emission-line knot results in radial velocities and FWHM that are consistent to within $\pm 50 \mathrm{~km} \mathrm{~s}^{-1}$. Furthermore, independent measurements of the same knots in both long-slit and slitless spectra yield values that agree to within $\pm 50 \mathrm{~km} \mathrm{~s}^{-1}$ (§2.1), so we adopt this as our typical uncertainity for both radial velocity and FWHM. The knots, with angular sizes of tenths of arcsecs, corresponding to tens of pcs, move coherently with their own peculiar motions, often superimposed on a more general flow. Although the amplitudes of the velocity curves differ significantly, there are a number of general trends.

Most of the Seyfert 1.9 and 2 galaxies in the sample show blueshifts and redshifts on either side of the nucleus, indicating that simple rotation is not the major source of the velocities. The only Seyfert 1 galaxy in the sample, NGC 3516, shows primarily blueshifts on one side of the nucleus and redshifts on the other side, like the Seyfert 1 galaxy NGC 4151 (Crenshaw et al. 2000). Taken together, these results support the biconical outflow 
picture, in which the bicone axis is near the plane of the sky for the Seyfert 2 galaxies and at an intermediate angle $\left(\sim 45^{\circ}\right)$ for the two Seyfert 1 galaxies with elongated emission, which results in one cone with approaching and the other with receding knots. One problem with this simple interpretation is that NGC 3516 shows "rogue" clouds moving in directions opposite to the general flow at $6^{\prime \prime}-7^{\prime \prime}$ from the nucleus, similar to that seen for a few low-intensity, high-velocity clouds in NGC 4151 (Hutchings et al. 1999; A. Das et al. 2004, in preparation). Another interesting case is the Seyfert 2 galaxy NGC 5728, which shows blueshifts on one side of its nucleus and redshifts on the other side. However, this could be matched with a biconical outflow model which is tilted significantly out of the plane of the sky, but not enough to allow us to see down the cone to the BLR.

The overall flow pattern in most objects is an increasing radial velocity from near zero at the nucleus to maximum blueshifts or redshifts within $0^{\prime \prime} .3-2^{\prime \prime}$ of the nucleus, followed by a more gradual decline to the systemic velocity. We have seen this trend before in NGC 1068 (Crenshaw \& Kraemer 2000) and NGC 4151 (Crenshaw et al. 2000, and reference therein). There are a few exceptions to this general trend. NGC 1386 has large blueshifts and redshifts at the nucleus and an unusual overall flow pattern. NGC 5728 has redshifted velocities at the nucleus, but it would fit the general trend if the center position were moved $\sim 0^{\prime \prime} .5$ to the west, coincident with the dust lane just above the nucleus in Figure 2. In Figure 4, NGC 7212 shows a mostly chaotic distribution of velocities with no clear pattern.

The plots in Figure 4 show an overall trend of decreasing FWHM with increasing distance from the nucleus, although in most cases there is a wide spread of values at each position. The bright nuclear knot of emission seen in every Seyfert galaxy in this sample except Mrk 3 has a large, and often the largest, FHWM, like that in NGC 4151 (Kaiser et al. 2000). The highest FWHMs tend to be comparable to the largest radial velocities in a Seyfert galaxy. On the other hand, Mrk 3 has several very bright knots of emission with large FWHMs near its nucleus, like NGC 1068 (Cecil et al. 2002). In these two Seyfert 2 galaxies, it may be that we are seeing expanded versions of the nuclear knots, since their central knots show large velocity dispersions and approximately zero radial velocity when averaged together (Crenshaw \& Kraemer 2000; Ruiz et al. 2001).

The nuclear emission-line knots in the majority of Seyfert galaxies consist of multiple kinematic components; they are not resolved spatially, but can be seen in spectral extractions around the nucleus. As an example, we show the nuclear spectrum of NGC 3081 in Figure 5. The extraction height perpendicular to the dispersion is $0^{\prime \prime} .15$, corresponding to the FWHM of the nuclear knot in the spatial direction. There are clearly two major kinematic components in the $\left[\mathrm{O}\right.$ III] $\lambda 5007$ line at $\sim-250$ and $\sim+50 \mathrm{~km} \mathrm{~s}^{-1}$. 


\section{Conclusions}

HST/STIS slitless spectroscopy of nearby Seyfert galaxies provides a useful snapshot of their NLR kinematics. The technique works when the NLR is clumpy and extended, so that individual emission-line knots can be identified in both direct and dispersed images. Our sample is therefore biased against NLRs that are smooth and/or compact, such as those typically found in Seyfert 1 galaxies (Schmitt et al. 2003b). The two Seyfert 1 galaxies that we have successfully mapped with slitless spectra, NGC 3516 and NGC 4151, have elongated NLRs. The disadvantage of the slitless technique is that we cannot measure the radial velocities of weak and/or smoothly distributed [O III] emission. Thus, long-slit spectra will often show a cleaner trend in the kinematics with position (e.g., compare the slitless measurements of Mrk 3 with the long-slit data in Ruiz et al. 2001). Nevertheless, we see clear trends in the radial velocities and FWHMs of emission-line knots as a function of projected radial distance from the optical continuum peaks in most of our objects. Trends as functions of distance or angle from the major axis of the NLR emission are weaker, if present, and likely require multiple long-slit observations to discern.

Most of the Seyfert 2 galaxies in our sample, along with NGC 1068 (Crenshaw \& Kraemer 2000) show significant blueshifts and redshifts on either side of their nuclei, which rules out simple rotation and is consistent with biconical outflow and the unified model, such that their bicone axes are relatively close to the plane of the sky. Our observations of the two Seyfert 1 galaxies NGC 3516 and NGC 4151 (Crenshaw et al. 2000) are also consistent with this picture; their elongated NLRs and the presence of blueshifts on one side of their nuclei and redshifts on the other indicate their bicone axes are at intermediate angles $\left(\sim 45^{\circ}\right)$ with respect to our lines of sight. The Seyfert 2 galaxy NGC 5728 appears to be viewed at an intermediate angle in the biconical outflow picture, but not enough for us to view the BLR directly.

Most of the Seyfert galaxies in our sample show a trend of increasing radial velocity to a projected angular distance of $0^{\prime \prime} .3-2^{\prime \prime}$, followed by a decrease to the systemic velocity further out. The radial acceleration could be due to radiation pressure or winds of more highly-ionized, diffuse gas. The source of the apparent deceleration is unknown, although one possibility is collision with an ambient medium (Crenshaw \& Kraemer 2000). The velocities in the ENLR are consistent with the finding that galactic rotation dominates the observed velocities (Unger et al. 1987), although the scatter is large, and we cannot rule out a secondary component of motion (such as radial outflow).

An interesting result from our slitless spectroscopy is that the emission-line knots, which have projected sizes of tens of pcs, are moving coherently, often with their own peculiar velocities. If they are indeed moving radially outward from the nucleus, their travel times 
out to several hundred pcs is on the order of $10^{5}$ to $10^{6} \mathrm{yr}$. It is interesting that the knots are able to maintain their integrity for this period of time, particularly since most have velocity dispersions on the order of several hundred $\mathrm{km} \mathrm{s}^{-1}$. The origin of the high dispersions (e.g., expansion, turbulence) is not known, and the reason for the decrease in FWHM with position is also unknown.

Finally, it is interesting that 10 out of 12 Seyfert galaxies that we have studied so far have a very bright, resolved knot of [O III] emission in their inner NLR, approximately centered on the optical continuum peak. These nuclear knots of emission also have large FWHMs, on the order of the velocities found in the intrinsic UV absorbers in Seyfert 1 galaxies (Crenshaw et al. 1999). A large fraction, and possibly most, of the UV absorbers are thought to be located at distance of tens of pcs from their central AGN (Crenshaw, Kraemer, \& George 2003), and it is tempting to associate them with the nuclear knots of emission. Further work is needed to test this possible association.

Support for proposal 8340 was provided by NASA through a grant from the Space Telescope Science Institute, which is operated by the Association of Universities for Research in Astronomy, Inc., under NASA contract NAS 5-26555. Some of the data presented in this paper were obtained from the Multimission Archive at the Space Telescope Science Institute (MAST). 


\section{REFERENCES}

Antonucci, R. 1993, ARA\&A, 31, 473

Axon, D.J., Marconi, A., Capetti, A., Macchetto, F.D., Schreier, E., \& Robinson, A. 1998, ApJ, 496, L75

Balkowski, C., Bottinelli, L., Gouguenheim, L., \& Heidmann, J. 1972, A\&A, 21, 303

Capetti, A., Axon, D.J., Macchetto, F.D., Marconi, A., \& Winge, C. 1999, ApJ, 516, 187

Cecil, G., Dopita, M.A., Groves, B., Wilson, A.S., Ferruit, P., Pecontal, E., \& Binette, L. 2002, ApJ, 568, 627

Crenshaw, D.M. and Kraemer, S.B. 2000, ApJ, 532, L101

Crenshaw, D.M., Kraemer, S.B., Boggess, A., Maran, S.P., Mushotzky, R.F., \& Wu, C.-C. 1999, ApJ, 516, 750

Crenshaw, D.M., Kraemer, S.B., \& George, I.M. 2003, ARA\&A, 41, 117

Crenshaw, D.M., Kraemer, S.B., Hutchings, J.B., Bradley, L.D.,II, Gull, T.R., Kaiser, M.E., Nelson, C.H., Ruiz, J.R., and Weistrop, D. 2000, AJ, 120, 1731

De Robertis, M.M., \& Shaw, R.A. 1990, ApJ, 348, 421

de Vaucouleurs, G., de Vaucouleurs, A., Corwin, H.G., Jr., Buta, R.J., Paturel, G., and Fouqué, P. 1991, Third Reference Catalog of Bright Galaxies (New York:Springer)

Dopita, M.A., Groves, B.A., Sutherland, R.S., Binette, L., \& Cecil, G. 2002, ApJ, 572, 753

Evans, I.N., et al. 1991, ApJ, 369, L27

Evans, I.N., et al. 1993, ApJ, 417, 82

Ferruit, P., Wilson, A.S., \& Mulchaey, J.S. 1998, ApJ, 509, 646

Huchtmeier, W.K., Richter, O.-G., 1989, A General Catalog of H I Observations of Galaxies, Springer Verlag

Hutchings, J.B., Crenshaw, D.M., Kaiser, M.E., Kraemer, S.B., Weistrop, D., Baum, S., Bowers, C.W., Feinberg, L.D., Green, R.F., Gull, T.R., Hartig, G.F., Hill, G., and Lindler, D.J. 1998, ApJ, 492, L115

Hutchings, J.B., Crenshaw, D.M., Danks, A.C., Gull, T.R., Kraemer, S.B., Nelson, C.H., Weistrop, D., Kaiser, M.E., \& Joseph, C.L. 1999, AJ, 118, 2101

Jaffe, W., et al. 2004, Nature, 429, 47

Kaiser, M.E., Bradley, L.D. II, Hutchings, J.B., Crenshaw, D.M., Gull, T.R., Kraemer, S.B., Nelson, C.H., Ruiz, J., and Weistrop, D. 2000, ApJ, 528, 260 
Kraemer, S.B. \& Crenshaw, D.M. 2000, ApJ, 544, 763

Malkan, M.A., Gorjian, V., \& Tam, R. 1998, ApJS, 117, 25

Moore, D. \& Cohen, R.D. 1996, ApJ, 470, 301

Nelson, C.H., Weistrop, D., Hutchings, J.B., Crenshaw, D.M., Gull, T.R., Kiaser, M.E., Kraemer, S.B., \& Lindler, D. 2000, ApJ, 531, 257

Nelson, C.H., \& Whittle, M. 1995, ApJS, 99, 67

Osterbrock, D.E. 1981, ApJ, 249, 462

Osterbrock, D.E. \& Mathews, W.G. 1986, ARA\&A, 24, 171

Peterson, B.M., et al. 2004, ApJ, in press (astro-ph/0407299)

Ruiz, J.R., Crenshaw, D.M., Kraemer, S.B., Bower, G.A., Gull, T.R., Hutchings, J.B., Kaiser, M.E. and Weistrop, D. 2001, AJ, 122, 2961

Schmitt, H.R., Donley, J.L., Antonucci, R.R.J., Hutchings, J.B., \& Kinney, A.L. 2003a, ApJS, 148, 327

Schmitt, H.R., Donley, J.L., Antonucci, R.R.J., Hutchings, J.B., Kinney, A.L., \& Pringle, J.E. 2003b, ApJ, 597, 768

Tifft, W.G., \& Cocke, W.J. 1988, ApJS, 67, 1

Unger, S.W., Pedlar, A., Axon, D.J., Whittle, M., Meurs, E.J.A., and Ward, M.J. 1987, MNRAS, 228, 671

Veilleux, S. 1991, ApJS, 75, 383

Winge, C., Axon, D.J., Macchetto, F.D., \& Capetti, A. 1997, ApJ, 487, L121

Winge, C., Axon, D.J., Macchetto, F.D., Capetti, A., \& Marconi, A. 1999, ApJ, 519, 134 
Fig. 1. - WFPC2 emission-line and continuum images and STIS slitless spectrum of the [O III] $\lambda 5007$ emission line in Mrk 3. The WFPC2 images have the same spatial scale and orientation as the STIS image. The "+" sign gives the location of the opical continuum peak.

Fig. 2.- HST emission-line images and STIS slitless spectra of the [O III] $\lambda 5007$ emission line in nine Seyfert galaxies. The WFPC2 images have the same spatial scale and orientation as the STIS image. The "+" signs give the locations of the opical continuum peaks. The diagonal bands in the NGC 5506 [O III] image are artifacts of the image rotation.

Fig. 3.- Radial velocity and FWHM of the [O III] emission as a function of angular distance from the optical continuum peak in Mrk 3. Negative and positive positions are below and above the central continuum peak in the image, respectively.

Fig. 4.- Radial velocity and FWHM of the [O III] emission as a function of angular distance from the optical continuum peak. Negative and positive positions are below and above the central continuum peaks in the images, respectively

Fig. 5.- Extracted spectrum from the nuclear [O III] knot in NGC 3081, plotted as a function of radial velocity relative to the systemic velocity of the host galaxy. 
Table 1. Sample

\begin{tabular}{lcccccc}
\hline \hline Object & $\begin{array}{c}\text { Seyfert } \\
\text { Type }^{\mathrm{a}}\end{array}$ & $\begin{array}{c}\text { Morph. } \\
\text { Type }\end{array}$ & $\begin{array}{c}\text { Redshift (cz) } \\
\left(\mathrm{km} \mathrm{s}^{-1}\right)\end{array}$ & $\begin{array}{c}\text { Redshift } \\
\text { Source }\end{array}$ & $\begin{array}{c}\text { Redshift } \\
\text { Reference }^{\mathrm{b}}\end{array}$ & $\begin{array}{c}\text { ENLR Shift } \\
\left(\mathrm{km} \mathrm{s}^{-1}\right)\end{array}$ \\
\hline Mrk 3 & 2 & S0 & $4050 \pm 6$ & $21 \mathrm{~cm} \mathrm{H} \mathrm{I}$ & TC & -116 \\
Mrk 573 & 2 & SB0 & $5150 \pm 11$ & Stellar & NW & +195 \\
Mrk 620 & 2 & SB(r)a & $1840 \pm 4$ & $21 \mathrm{~cm} \mathrm{H} \mathrm{I}$ & $3 \mathrm{RC}$ & 0 \\
NGC 1386 & 2 & SB0 & $918 \pm 34$ & $21 \mathrm{~cm} \mathrm{H} \mathrm{I}$ & HR & -88 \\
NGC 3081 & 2 & SB0 & $2367 \pm 9$ & $21 \mathrm{~cm} \mathrm{H} \mathrm{I}$ & $3 R C$ & +92 \\
NGC 3516 & 1 & SB0 & $2503 \pm 75$ & $21 \mathrm{~cm} \mathrm{H} \mathrm{I}$ & B & -66 \\
NGC 5506 & 1.9 & Sa & $1815 \pm 9$ & $21 \mathrm{~cm} \mathrm{H} \mathrm{I}$ & $3 R C$ & 0 \\
NGC 5643 & 2 & SABc & $1199 \pm 5$ & $21 \mathrm{~cm} \mathrm{H} \mathrm{I}$ & $3 R C$ & 0 \\
NGC 5728 & 2 & SAB(r)a & $2788 \pm 8$ & $21 \mathrm{~cm} \mathrm{H} \mathrm{I}$ & $3 R C$ & 0 \\
NGC 7212 & 2 & Sa & $8011 \pm 21$ & Stellar & NW & +102 \\
\hline
\end{tabular}

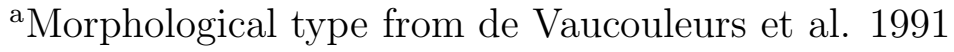

${ }^{\text {b}}$ TC- Tifft \& Cooke (1988), 3RC- de Vaucouleurs et al. (1991), NW - Nelson \& Whittle (1995), HR - Huchtmeier \& Richter (1989), B - Balkowski et al. (1972)

${ }^{\mathrm{c}}$ Correction added to the radial velocities to force the average ENLR velocity to be zero (see §2.3.) 
Table 2. STIS Observations of Seyfert Galaxies

\begin{tabular}{lccccc}
\hline \hline Galaxy & $\begin{array}{c}\text { STScI } \\
\text { Dataset }\end{array}$ & $\begin{array}{c}\text { Date } \\
(\mathrm{UT})\end{array}$ & $\begin{array}{c}\text { Central } \lambda \\
(\AA)\end{array}$ & $\begin{array}{c}\text { Exposure } \\
(\mathrm{s})\end{array}$ & $\begin{array}{c}\text { PA } \\
\left({ }^{\circ}\right)\end{array}$ \\
\hline Mrk 3 & O5F403020 & 2000 Jan 16 & 5093 & 2154 & 112 \\
Mrk 573 & O5F401020 & 2000 Oct 24 & 5093 & 2096 & 330 \\
Mrk 620 & O5F404020 & 2000 Mar 3 & 4961 & 2128 & 50 \\
NGC 1386 & O5F402020 & 2000 Jun 23 & 4961 & 2106 & 175 \\
NGC 3081 & O5F405020 & 2000 Feb 15 & 5093 & 2108 & 335 \\
NGC 3516 & O5F406020 & 2000 Jan 18 & 5093 & 2154 & 205 \\
NGC 5506 & O5F407020 & 2000 Mar 18 & 4961 & 2096 & 206 \\
NGC 5643 & O5F408020 & 2000 Feb 23 & 4961 & 2107 & 260 \\
NGC 5728 & O5F409020 & 2000 Apr 24 & 5093 & 2110 & 280 \\
NGC 7212 & O5F410020 & 2000 Jun 4 & 5093 & 2112 & 185 \\
\hline
\end{tabular}


Table 3. HST Archival Images

\begin{tabular}{lcccccc}
\hline \hline Galaxy & $\begin{array}{c}\text { STScI } \\
\text { Dataset }\end{array}$ & $\begin{array}{c}\text { Date } \\
(\mathrm{UT})\end{array}$ & Camera & Filter & $\begin{array}{c}\text { Exposure } \\
(\mathrm{s})\end{array}$ & $\begin{array}{c}\text { PA } \\
\left({ }^{\circ}\right)\end{array}$ \\
\hline \multirow{2}{*}{ Mrk 3 } & W0MW0601T & 1991 July 18 & PC & F502N & 1800 & 151 \\
& X14W0301T & 1992 Dec 11 & FOC & F501N & 1197 & 344 \\
& W0MW0602T & 1991 July 18 & PC & F547M & 350 & 151 \\
& U2E62A01T & 1994 Oct 20 & WFPC2 & F606W & 500 & 298 \\
Mrk 573 & U2XI0701T & 1995 Nov 12 & WFPC2 & FR533N & 300 & 214 \\
& X2580504T & 1994 Feb 9 & FOC & F550M & 1300.9 & 39 \\
Mrk 620 & U3A00301T & 1997 Feb 5 & WFPC2 & FR533N & 800 & 267 \\
& U3A00303T & 1997 Feb 5 & WFPC2 & F547M & 100 & 267 \\
NGC 1386 & U3A00201M & 1997 June 28 & WFPC2 & F502N & 400 & 266 \\
& U3A00203M & 1997 June 28 & WFPC2 & F547M & 40 & 266 \\
NGC 3081 & U3A00501T & 1997 Feb 5 & WFPC2 & FR533N & 200 & 119 \\
& U3A00503T & 1997 Feb 5 & WFPC2 & F547M & 40 & 119 \\
NGC 3516 & U3A00602T & 1997 Feb 7 & WFPC2 & FR533N & 800 & 343 \\
& U3A00603T & 1997 Feb 7 & WFPC2 & F547M & 70 & 343 \\
NGC 5506 & X2740302T & 1995 Jan 29 & FOC & F501N & 1797 & 255 \\
& U2E62001T & 1994 July 21 & WFPC2 & F606W & 500 & 155 \\
& X2740301T & 1995 Jan 29 & FOC & F550M & 1996 & 255 \\
NGC 5643 & U2NP0401T & 1995 June 2 & WFPC2 & F502N & 350 & 106 \\
& U2NP0403T & 1995 June 2 & WFPC2 & F547M & 50 & 106 \\
NGC 5728 & W1150401T & 1992 Sept 4 & PC & F492M & 600 & 288 \\
& W1150403T & 1992 Sept 4 & PC & F547M & 600 & 288 \\
NGC 7212 & U2XI0401T & 1995 Sept 26 & WFPC2 & FR533N & 300 & 248 \\
& U2XI0403T & 1995 Sept 26 & WFPC2 & FR533N & 140 & 248 \\
& U2E65301T & 1994 Sept 27 & WFPC2 & F606W & 500 & 160 \\
\hline
\end{tabular}




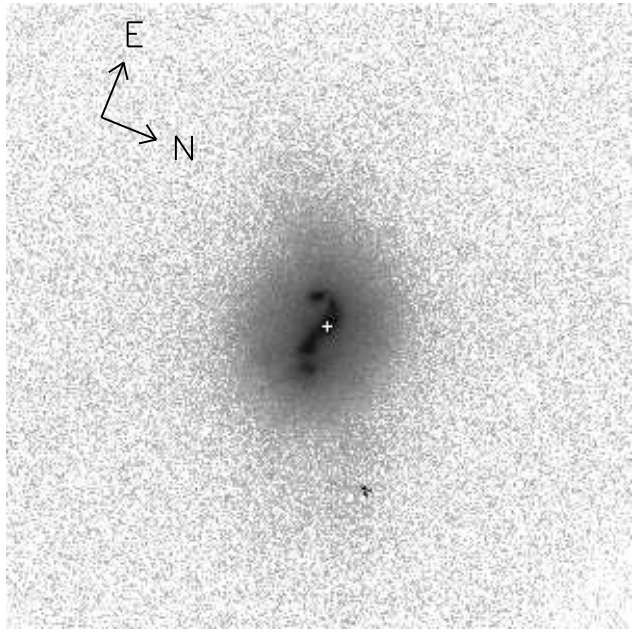

Mrk 3 WF/PC F502N [O III]

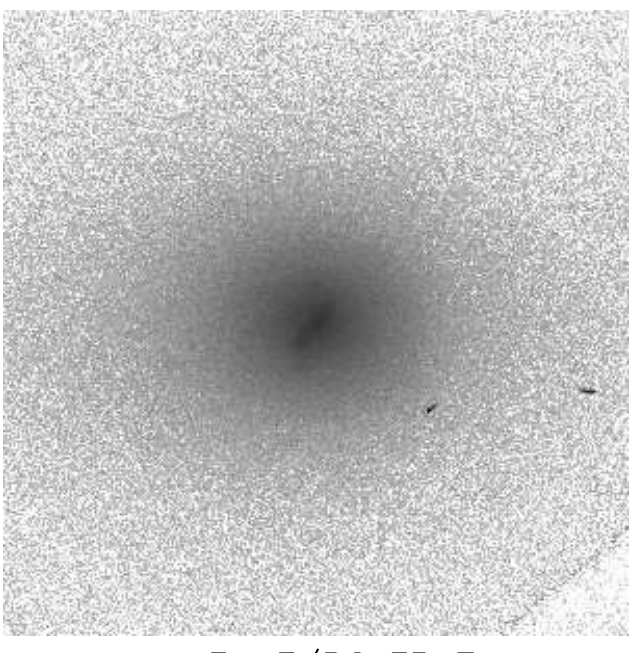

Mrk 3 WF/PC F547M

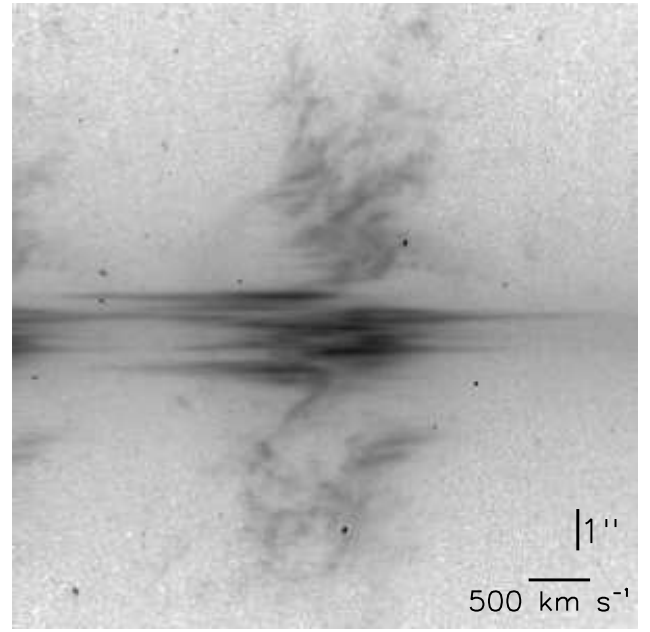

Mrk 3 STIS Slitless

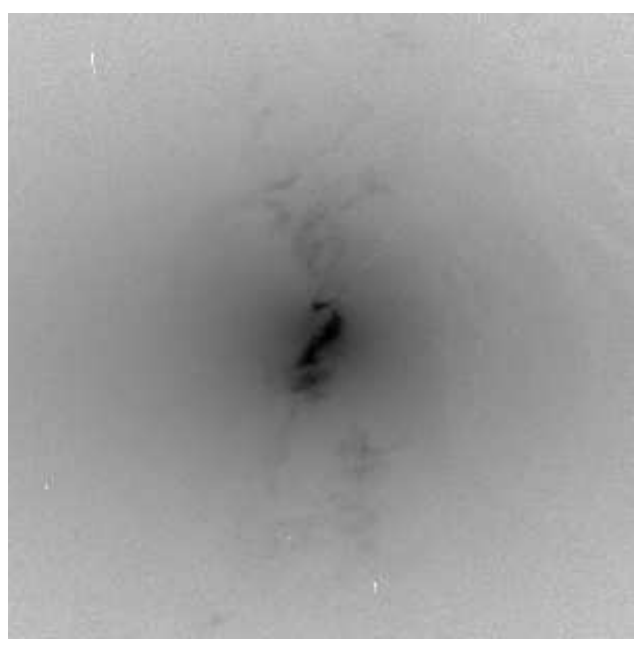

Mrk 3 WFPC2 F606W

Fig. 1. 


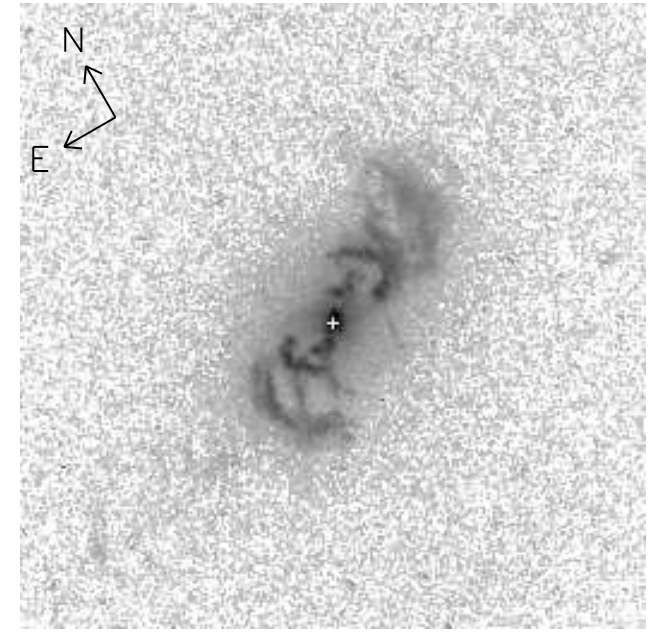

Mrk 573 WFPC2 [0 III]

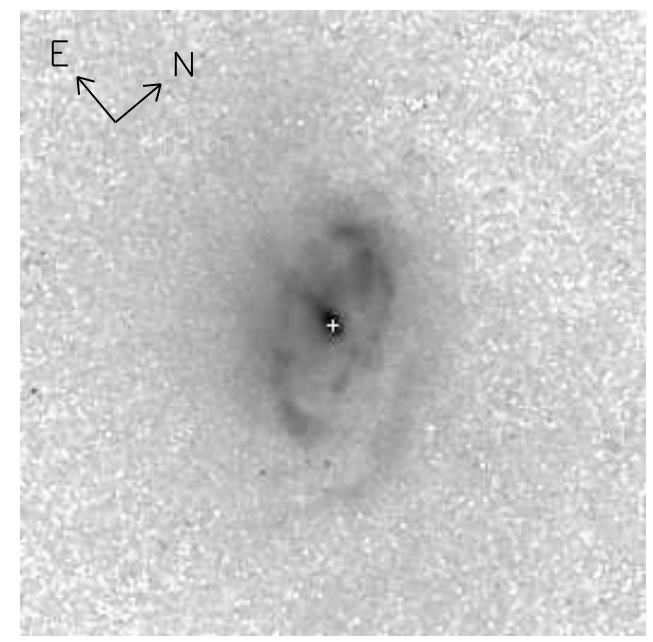

Mrk 620 WFPC2 [O III]

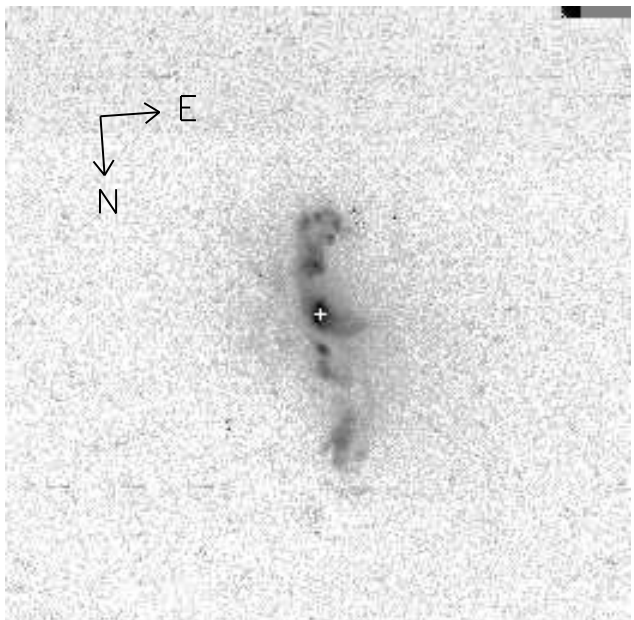

NGC 1386 WFPC2 [O III]

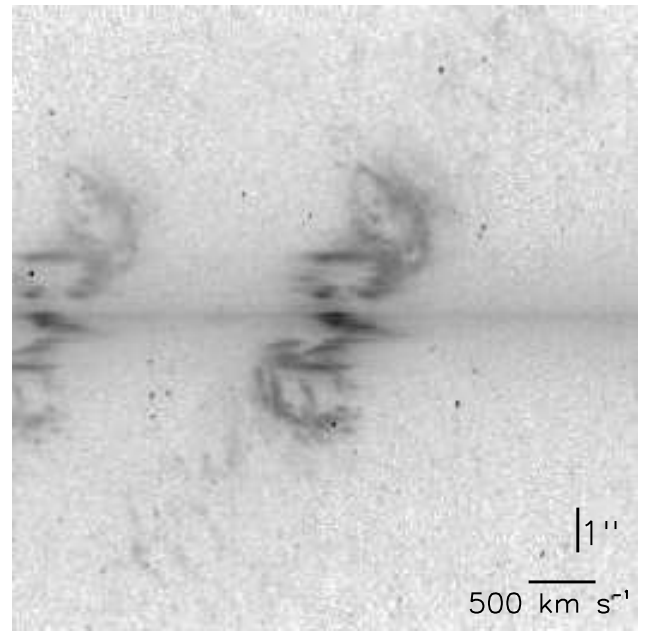

Mrk 573 STIS Slitless

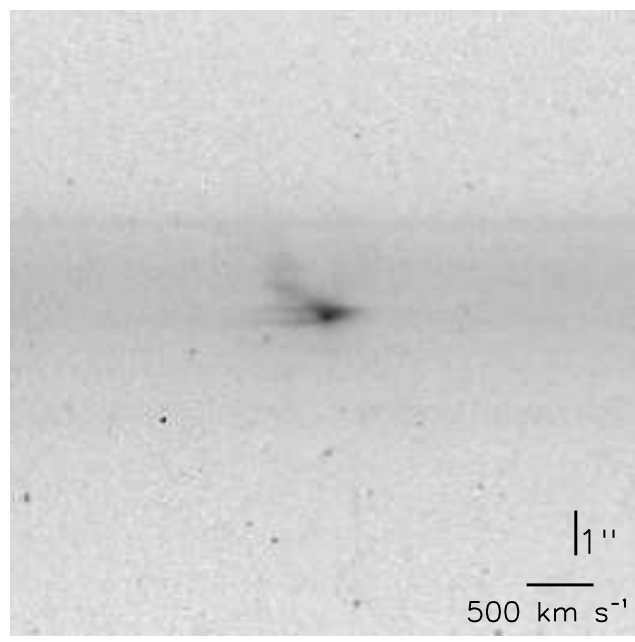

Mrk 620 STIS Slitless

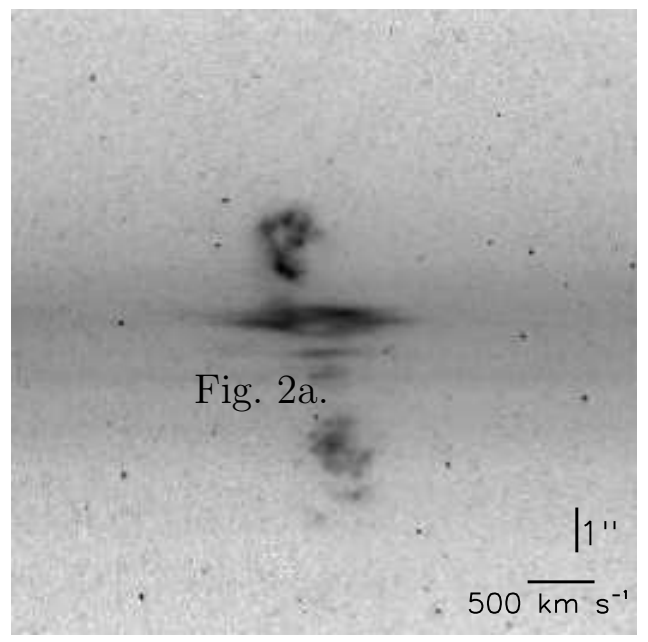

NGC 1386 STIS Slitless 

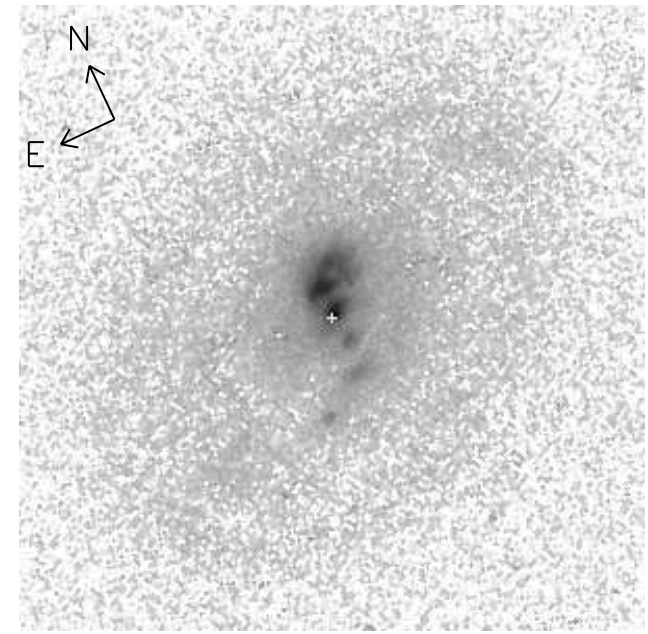

NGC 3081 WFPC2 [0 III]

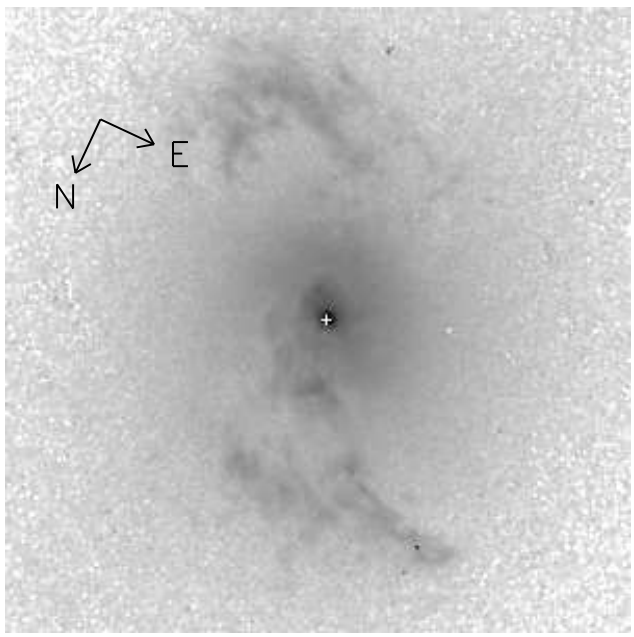

NGC 3516 WFPC2 [O III]
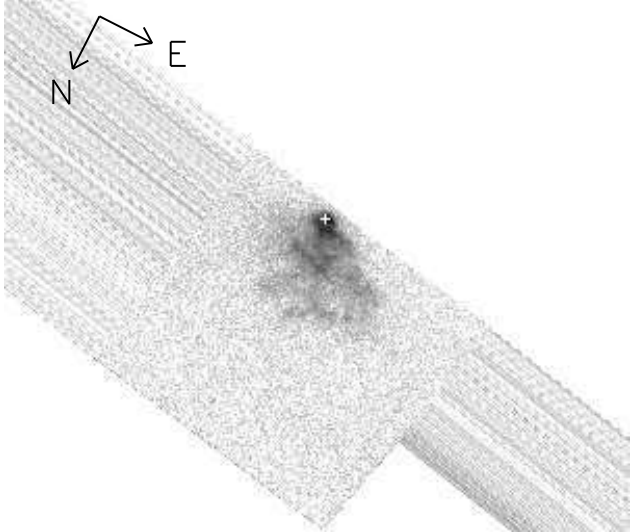

NGC 5506 FOC [O III]

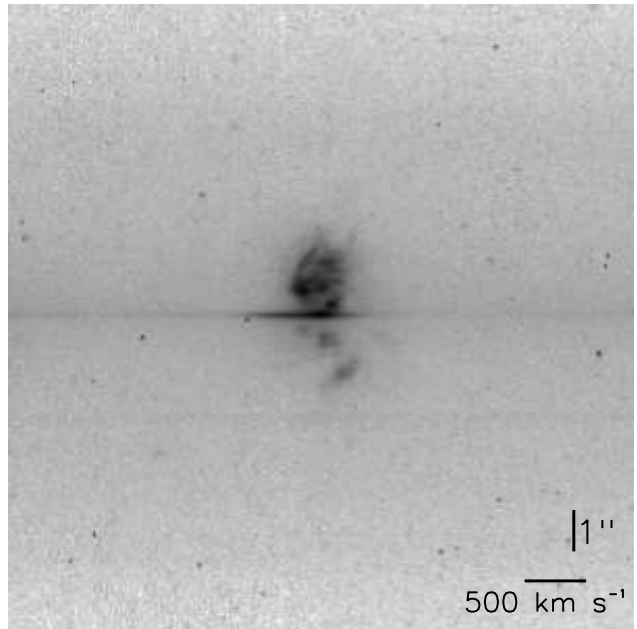

NGC 3081 STIS Slitless

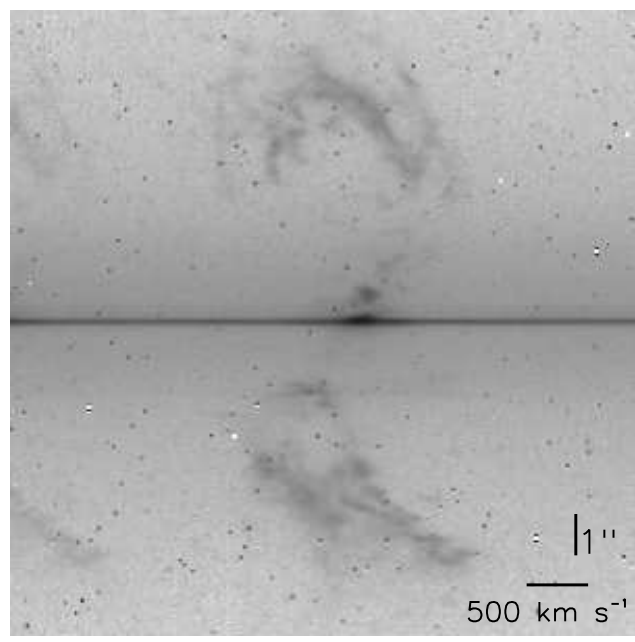

NGC 3516 STIS Slitless

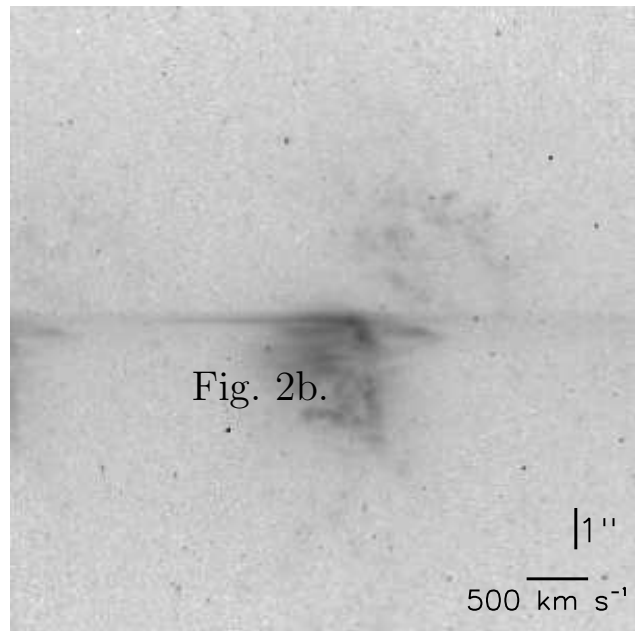

NGC 5506 STIS Slitless 

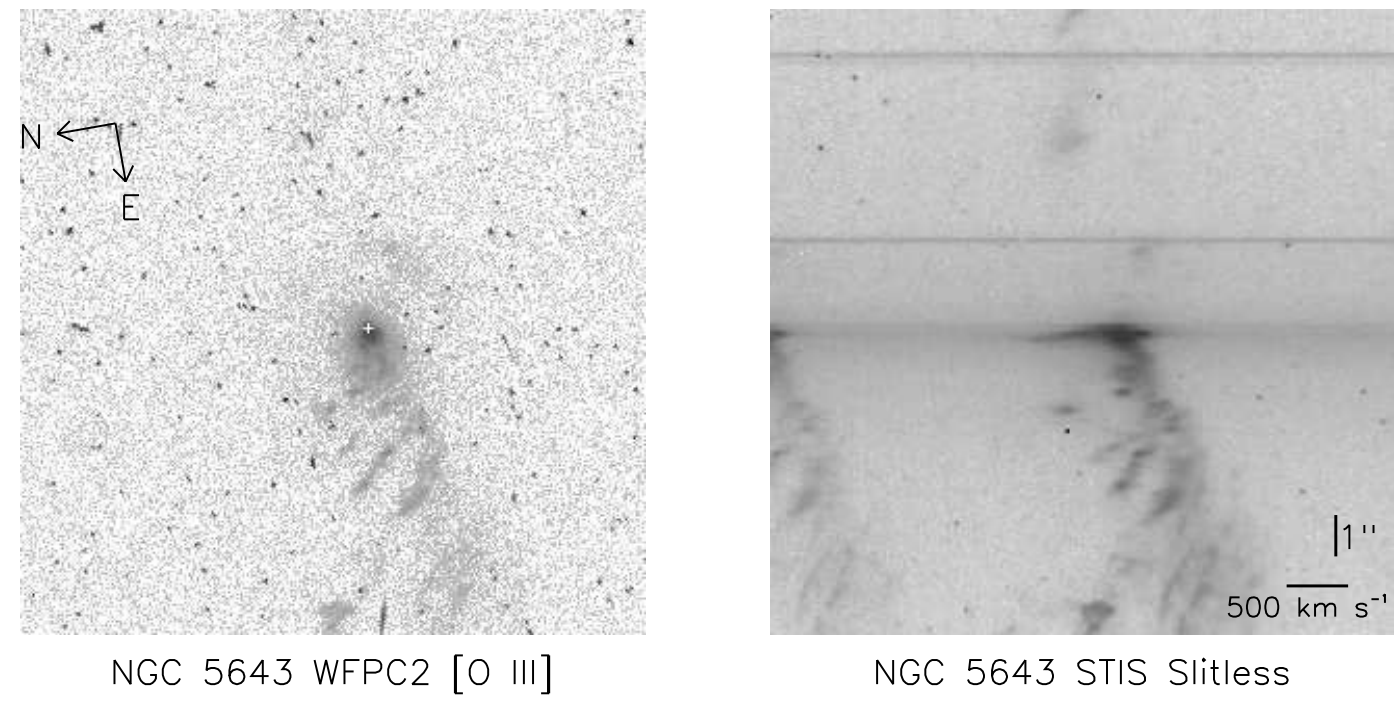

NGC 5643 STIS Slitless
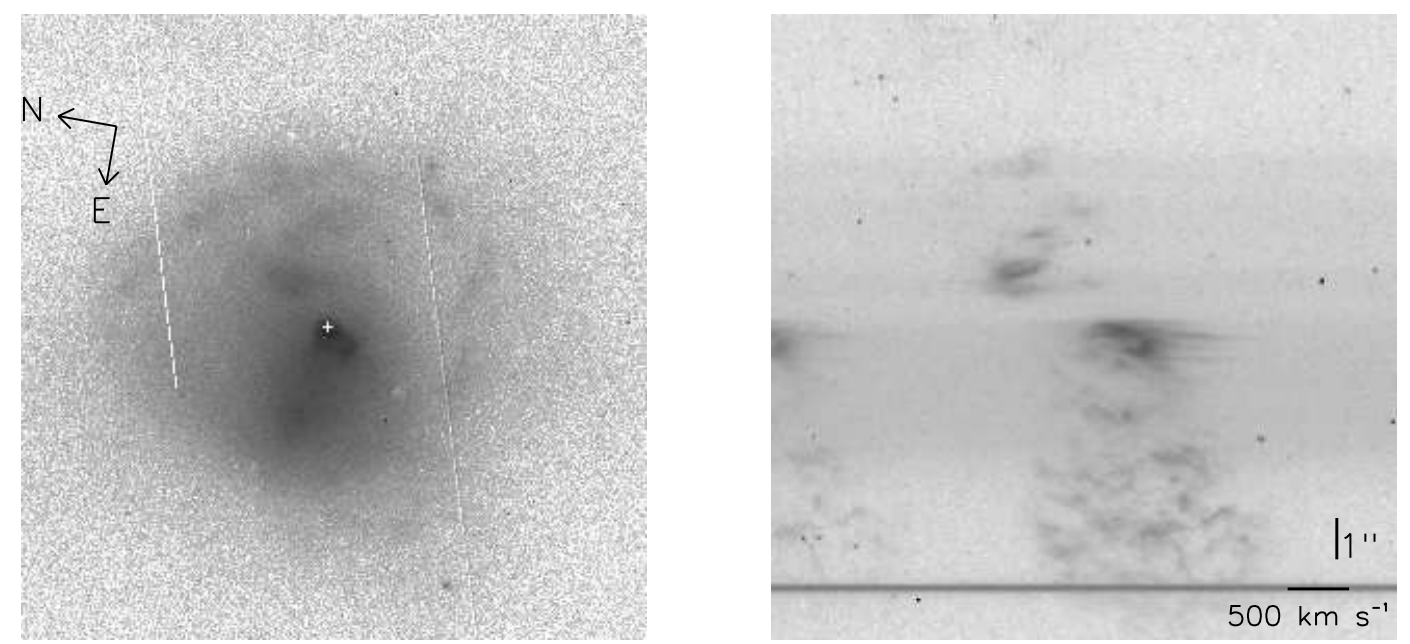

NGC 5728 WF/PC [O III]

NGC 5728 STIS Slitless
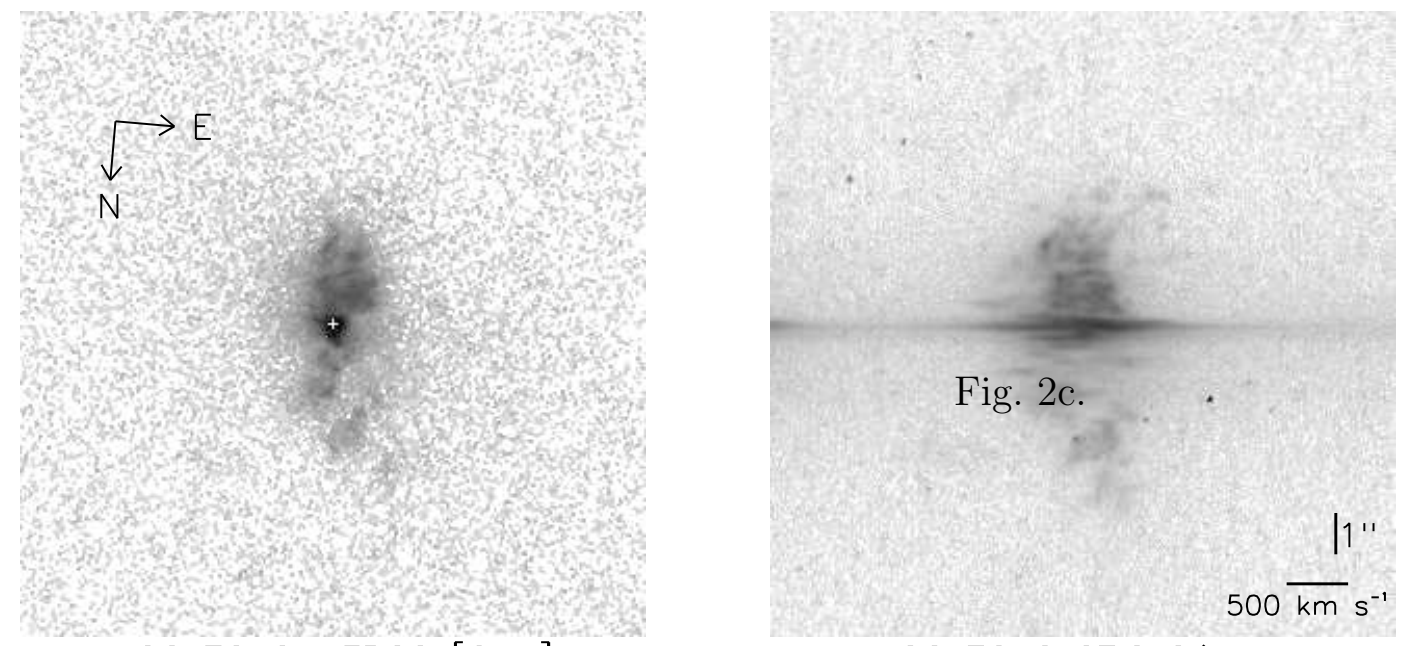

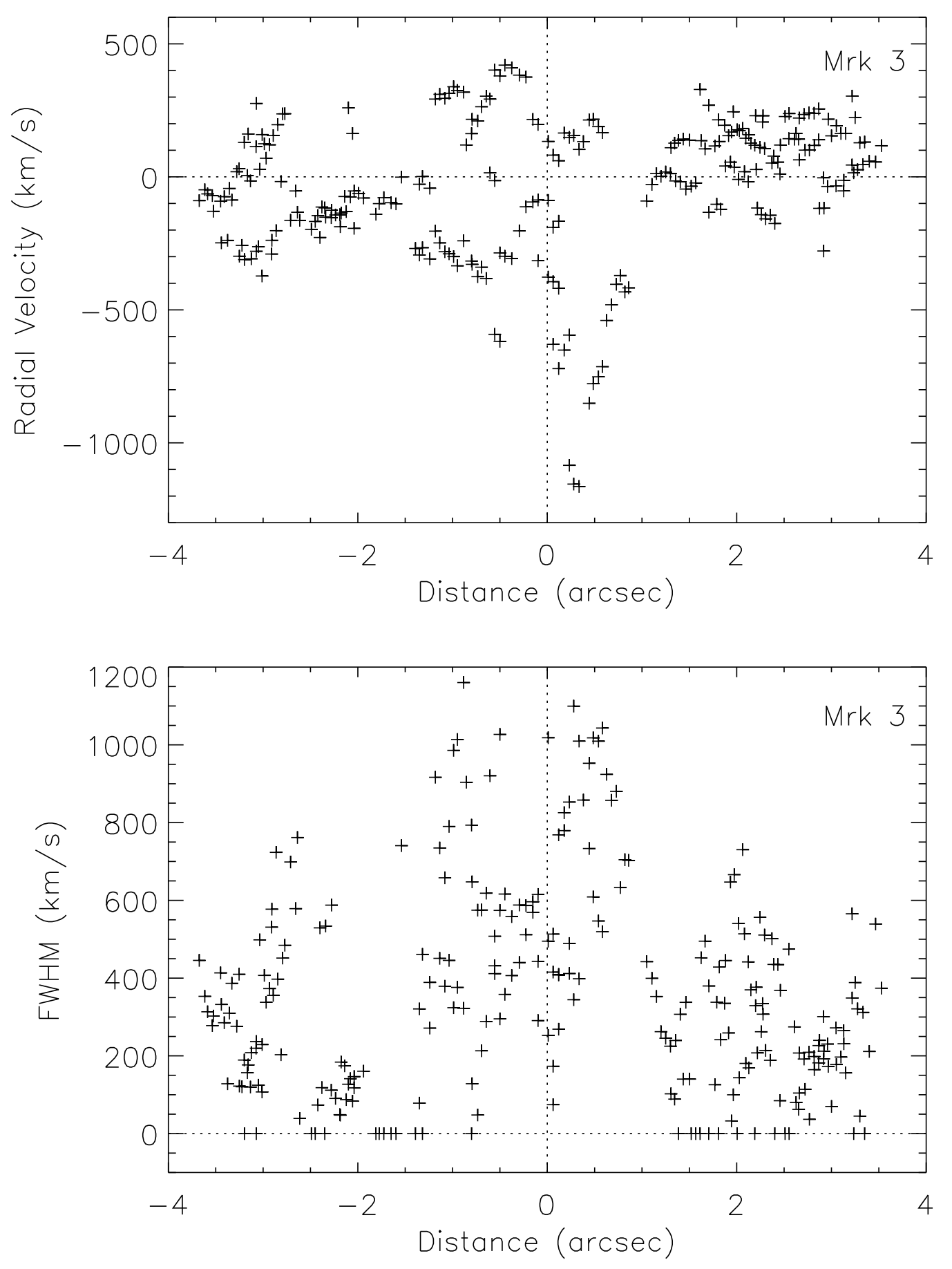

Fig. 3. 

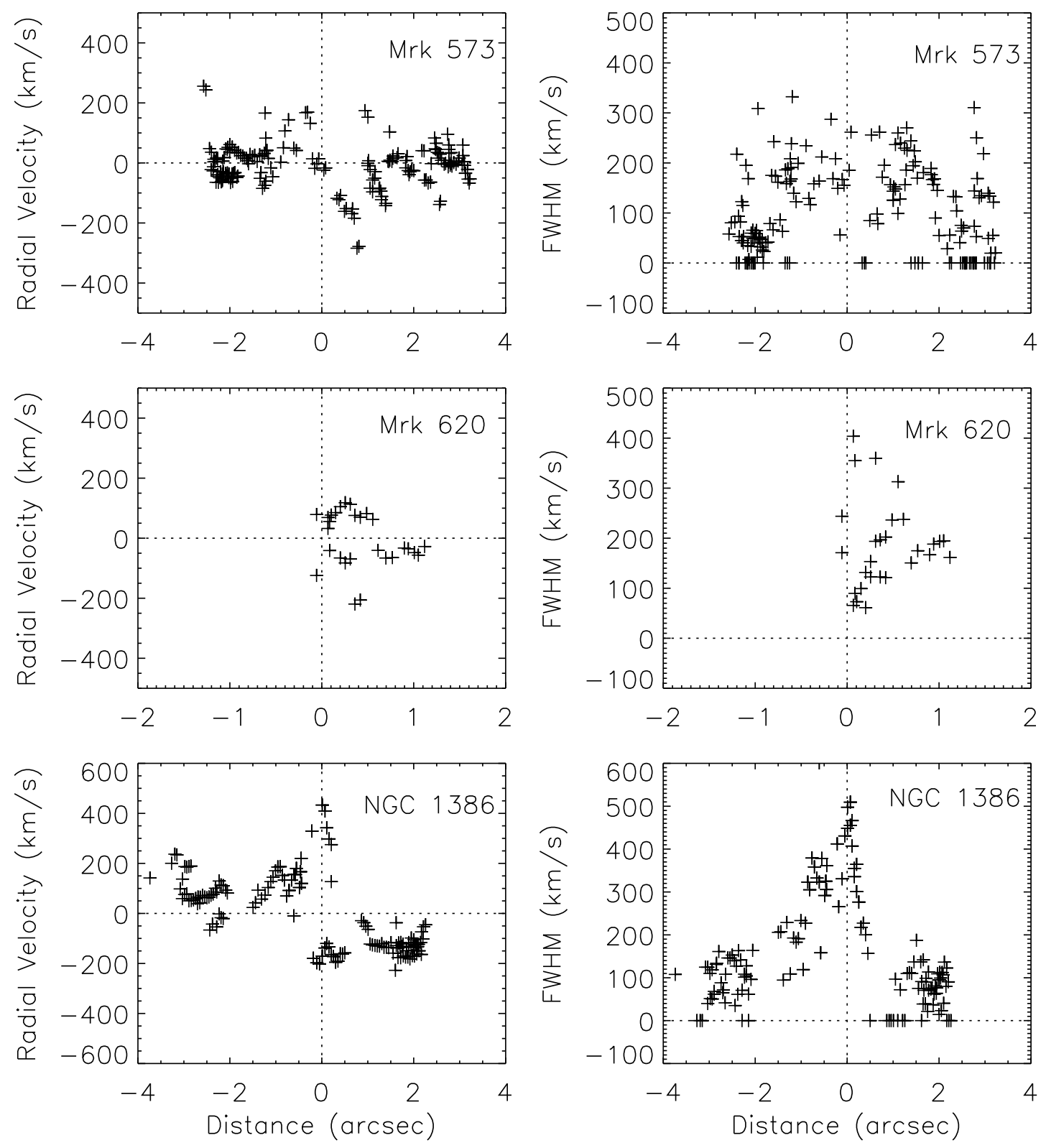

Fig. 4a. 

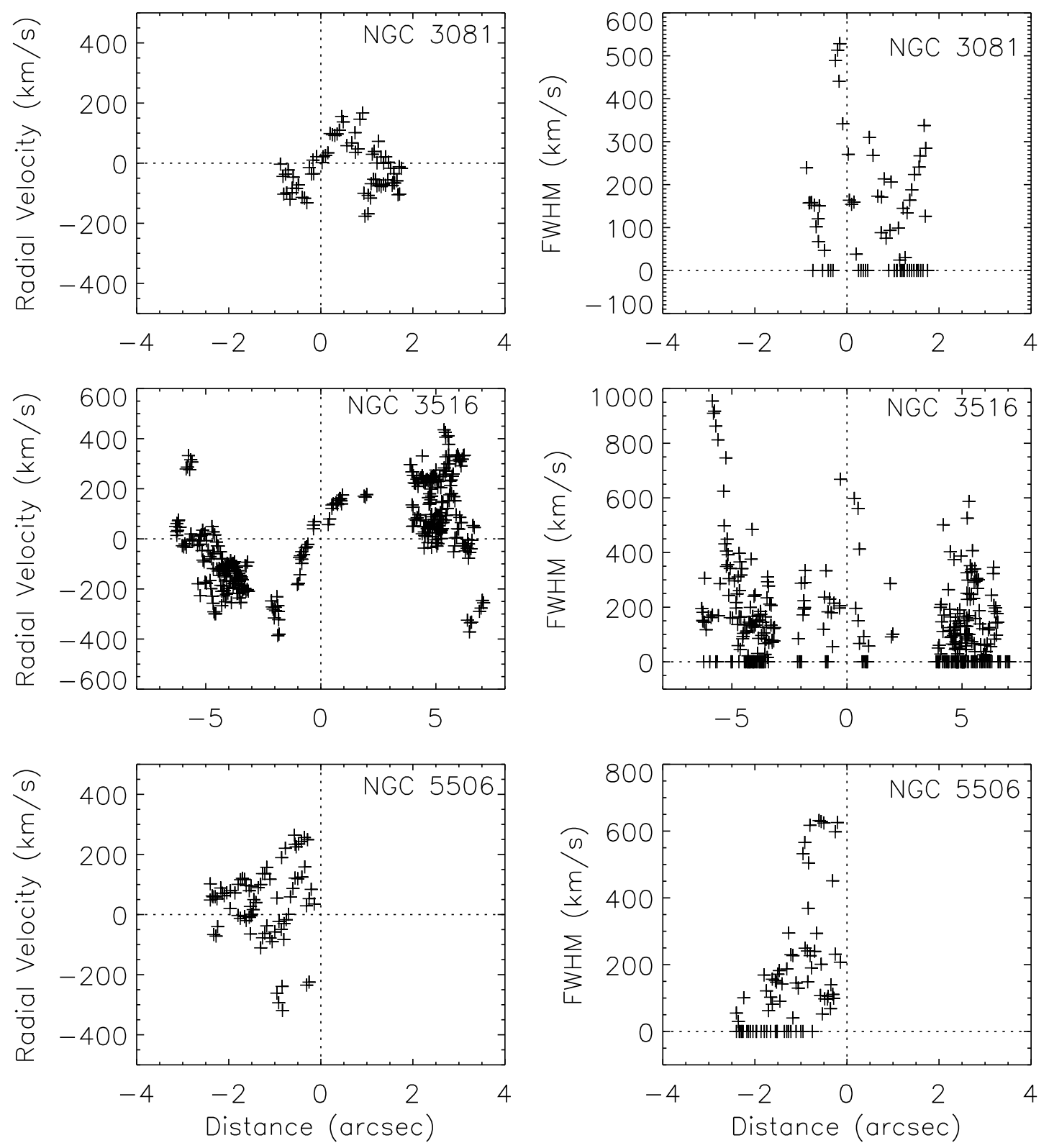

Fig. 4b. 

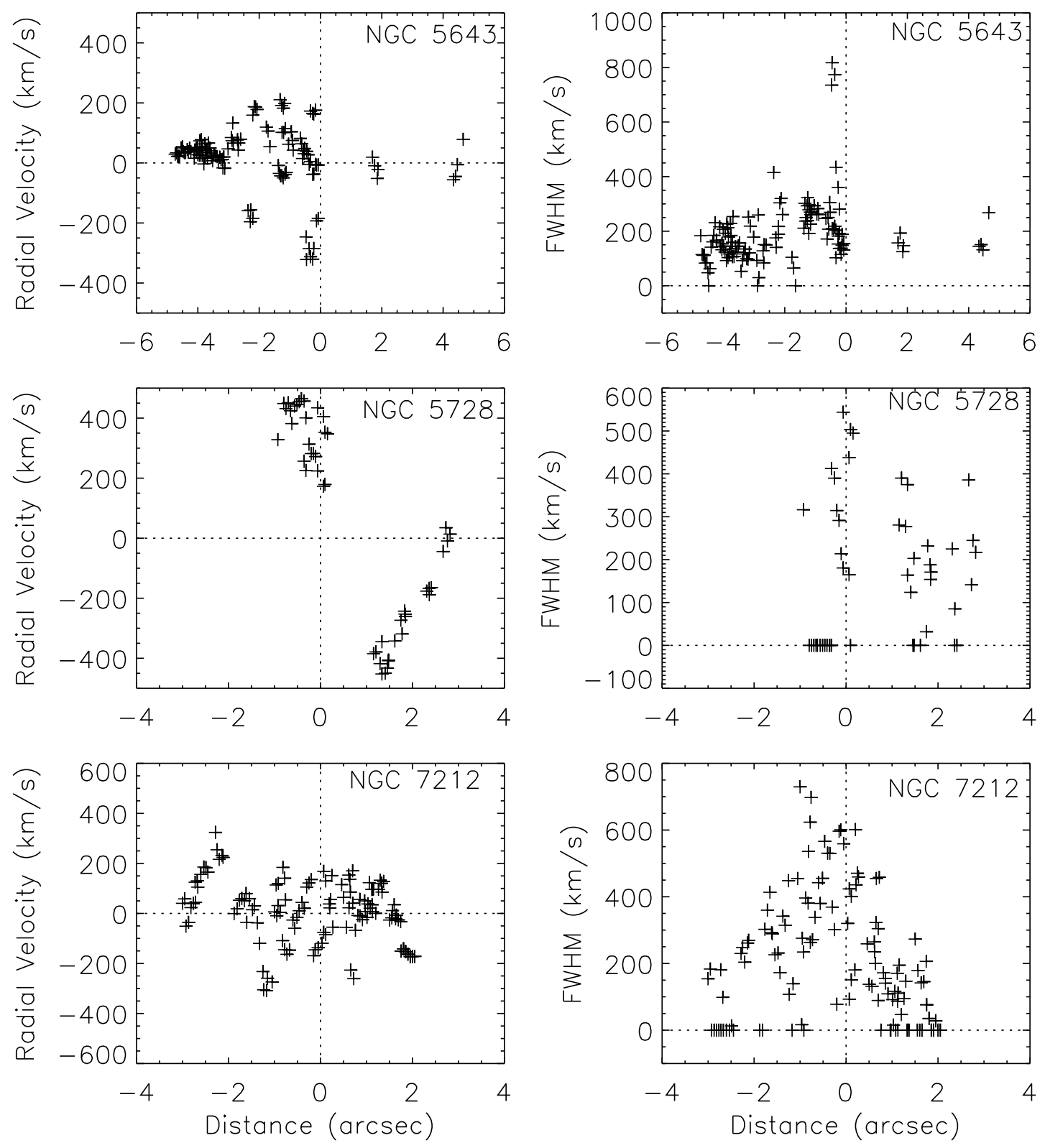

Fig. 4c. 


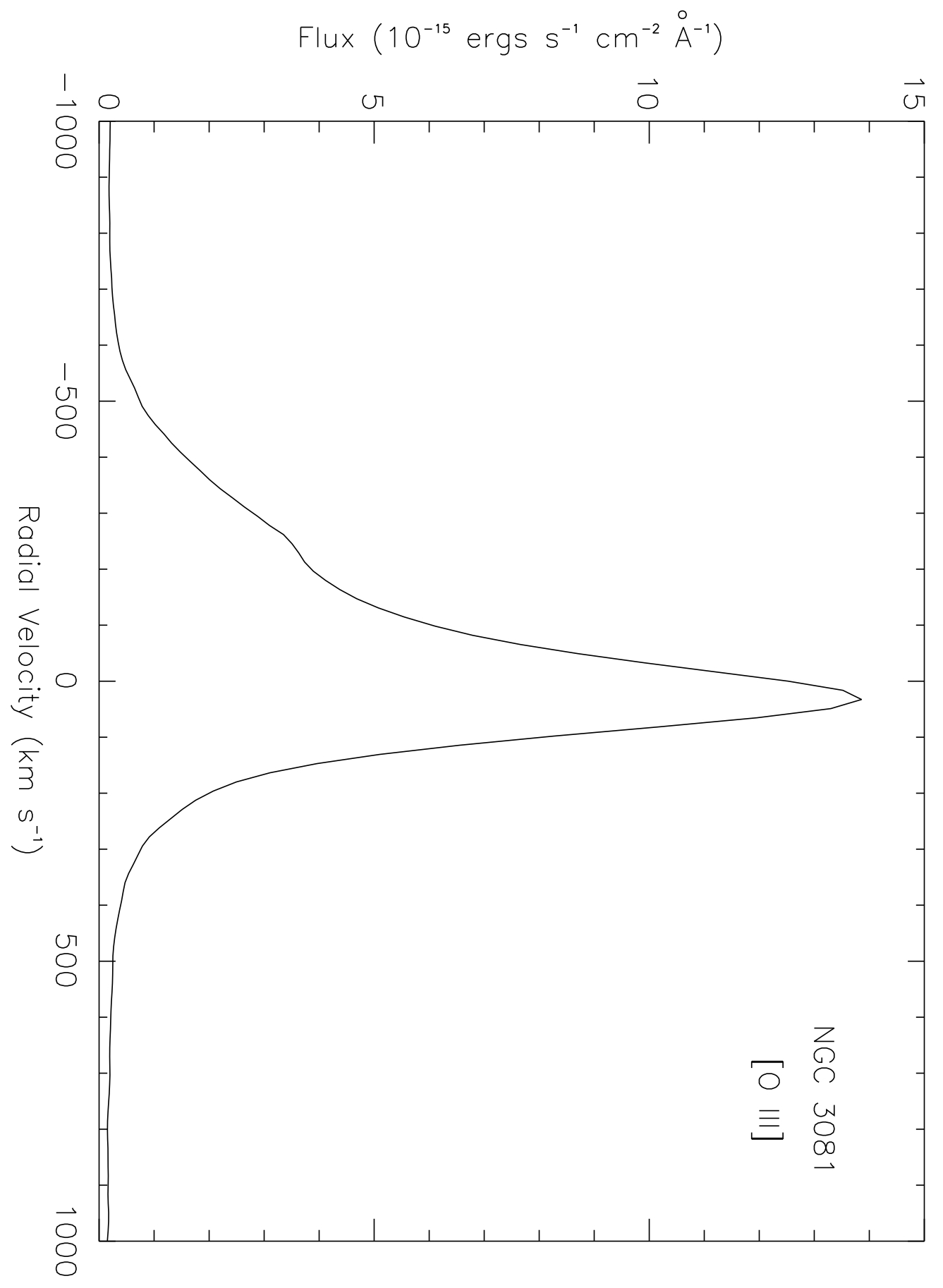

Fig. 5. 